\title{
Should the subventricular zone be part of the "rad" zone?
}

\author{
Benjamin W. Corn · Jeffrey Raizer • \\ Andrew A. Kanner
}

Received: 13 March 2014/ Accepted: 23 April 2014/Published online: 23 May 2014

(C) Springer Science+Business Media New York 2014

\section{Editorial}

In American football, the "red zone" refers to an area of the playing field adjacent to the goal line where the chances of scoring are statistically higher. In the discipline of geography, the term refers to a region of decimation which typically follows natural disasters (e.g., earthquakes) or military invasion (e.g. Zone Rouge in France in the aftermath of World War I). By analogy, a "rad zone," might be thought of as a region where high doses of irradiation are deposited in order to decimate malignant cells with the intent of bringing about statistically higher rates of tumor control.

In the war on glioblastoma (GBM), perhaps the most lethal malignancy that arises in the central nervous system, a debate has been raging during the last 2 years regarding the importance of a different zone-the subventricular zone (SVZ). That region, which is located under the ependyma of the lateral ventricles, is a critical niche for neurogenesis in the adult human brain. With the publication by Elicin et al. [1] in this edition of The Journal, contradictory data now exist regarding the prudence of delivering high doses of radiation therapy within this

B. W. Corn $(\bowtie)$

Institute of Radiotherapy, Tel Aviv Medical Center, Tel Aviv University School of Medicine, 6 Weizman Street, Tel Aviv, Israel

e-mail: bencorn@tlvmc.gov.il

J. Raizer

Department of Neurology, Northwestern University, Chicago, IL, USA

\section{A. A. Kanner}

Stereotactic Radiosurgery Unit, Department of Neurosurgery, Tel Aviv Sourasky Medical Center, Tel Aviv University,

Tel Aviv, Israel ostensible source of glioma cells. The time has come for radiation oncologists with an interest in brain tumors to decide whether to make the subventricular zone and the rad zone coincide.

Traditionally, the SVZ has not been part of the lexicon for the radiation treatment planning of GBM. Indeed, "clinical target volumes" have been influenced by classic papers that used 3-dimensional imaging [2,3] or postmortem examination [4] to add concentric isodose clouds around contrast enhancing abnormalities and or edema in treatment that is delivered either in one stage (e.g., EORTC) or via some variant of the so-called "shrinking field technique" (e.g. RTOG). If the SVZ is to become part of the CTV, then a dogmatic paradigm is shifting before our eyes. Suddenly, radiation oncologists may be called upon to expand their fields beyond the implicit existence of microscopic disease juxtaposed to the obvious focus of tumor. Moreover, instead of seeking out a downstream station of disease - as is typically done in nodal irradiation for lymphoma and carcinomas arising below the base of skull-here the idea would be to suppress pools of relatively remote precursor cells that presumably replenish the tumor mass.

Elicin et al. meticulously analyzed a small group $(\mathrm{N}=60)$ of patients with GBM who were irradiated to $60 \mathrm{~Gy}$ in a single phase. In a retrospective assessment of cSVZ dose, the authors noted that patients receiving $>59.2$ Gy had a statistically inferior median progressionfree survival time when compared to those receiving lower doses to the same region. The investigators inferred that their findings were not a proxy for volume effect since the extent of the CTV itself was not associated with PFS or OS in either univariate or multivariate analyses.

Although there are caveats when progression free survival is used as an endpoint for GBM [5], students of the 
evolving literature of SVZ dose in the irradiation of glioblastoma will immediately recognize the importance of the report from Elicin et al. since this is not really a neutral study but one that suggests that high dose deposition in the SVZ could be deleterious. The contrast to previous articles dedicated to the subject is stark. For instance, Evers et al. [6] noted that patients whose bilateral sub-ventricular zones received greater than the median SVZ dose had a significant improvement in progression free survival in comparison to patients who received less than the median dose (15.0 vs. 7.2 months PFS; $P=0.028)$. Similarly, a combined analysis from Johns Hopkins and Duke [7] showed that high doses to the ipsilateral SVZ (mean $\geq 40 \mathrm{~Gy}$ ) among patients whose glioblastoma was gross totally resected, was associated with significant improvements in PFS and OS. In a study from Japan, where hypofractionated IMRT was used with concurrent and adjuvant temozolomide for GBM, Iuchi et al. [8] noted that necrosis in the ipsilateral SVZ was significantly correlated with not only prolonged survival but also deterioration in the performance status of those who survived long-term.

Indeed the harmful impact of treating the SVZ is not surprising since neural stem cell compartments have been implicated in neurocognitive decline due to hints of memory preservation observed when conformal avoidance strategies were designed to shield the dentate gyrus of the hippocampus among patients referred for whole brain irradiation $[9,10]$. Can the new neurobiology be selectively harnessed to protect patients from toxicity in the case of brain metastases and to augment the control of a devastating entity like GBM? In theory, and as Elicin et al. advocate in their Discussion, carefully designed prospective randomized trials are needed to answer such questions.

To make therapeutic advances we will need to wisely approach the challenge as we seek to reconcile the conflicting data and determine the true impact of SVZ irradiation. Until then, other institutions-or groups of institutions willing to pool data-should be encouraged to review their experiences with SVZ dosimetry (preferably without arbitrary dose cutoffs) among patients treated for glioblastoma. Attention should be paid to the ipsilateral as well as contralateral subventricular zones. To what extent is "symptom burden" increased if larger volumes of brain are irradiated? And will larger volumes exacerbate lymphopenia [11] and thereby offset theoretical survival advantages attributable to treatment of the SVZ?

Going forward, investigators should also account for the methylation status of the MGMT promoter region, since this important discriminant has not been rigorously included in the multivariate analyses performed to date. In addition, consistency must be sought among neuro-oncologists in distinguishing between pseudoprogression and frank progression in generating a hypothesis that is largely predicated on assessment of failure patterns. Even more rudimentary, how do we delineate the SVZ itself? Is it a discreet anatomic compartment that we can define with atlases, a functional construct or a little of both? Should we be altering our concept of the SVZ because of presumed modification by surgical resection or cytotoxic therapies aside from irradiation? And, ideally, before significant resources are expended, can we identify a surrogate endpoint that might constitute a putative biomarker to help us understand mechanism and fine tune interventions like radiation dose prescription or even to determine whether there may be a role for inhibitors directed against tumor initiating cells?

In American football, it is axiomatic that despite the proximity of the red zone to the goal line, it is no simple matter to, in fact, score. Teams who invest the effort to map out sound strategies often emerge victoriously. Rather than cavalierly concluding that the SVZ must be contained within the rad zone, the opportunity now exists for the respective players on the neuro-oncology team to devise a sound game plan for unraveling the intriguing riddle of the subventricular zone.

\section{References}

1. Elicin O, Inac E, Uzel EK, Karacam S, Uzel OE (2014) Relationship between survival and increased radiation dose to subventricular zone in glioblastoma is controversial. J Neurooncol. doi:10.1007/s11060-014-1424-3

2. Hochberg FH, Pruitt A (1980) Assumptions in the radiotherapy of glioblastoma. Neurology 30:907-911

3. Earnest F, Kelly PJ, Scheithauer BW et al (1988) Cerebral astrocytomas: histopathologic correlation of MR and CT contrast enhancement with stereotactic biopsy. Radiology 166:823-827

4. McComb RD, Burger PC (1985) Pathologic analysis of primary brain tumors. Neurol Clin 3:711-728

5. Fine HA (2014) Bevacizumab in glioblastoma—still much to learn. N Engl J Med 370:764-765

6. Lee P, Eppinga W, Lagerwaard F et al (2013) Evalulation of high ipsilateral subventricular zone radiatin therapy dose in glioblastoma: a pooled analysis. Int J Radiat Oncol Biol Phys 86:609-615

7. Chen L, Guerrero-Cazares H, Ye X et al (2013) Increased subventricular zone radiation dose correlates with survival in glioblastoma patients after gross total resection. Int $\mathrm{J}$ Radiat Oncol Biol Phys 86:616-622

8. Iuchi T, Hatano K, Kodama T, et al. (2014) Phase 2 trial of hypofractionated high-dose intensity modulated radiation therapy with concurrent and adjuvant temozolomide for newly diagnosed glioblastoma. Int J Radiat Oncol Biol Phys 88:793-800

9. Bannerman DM, Sprengel R, Sanderson DJ et al (2014) Hippocampal synaptic plasticity, spatial memory and anxiety. Nature Rev 15:181-192

10. Gondi V, Mehta MP, Pugh S et al (2013) Memory preservation with conformal avoidance of the hippocampus during whole brain radiotherapy for patients with brain metastases: Results of RTOG 09-33. ASTRO, Plenary Session

11. Holdhoff M (2011) Grossman SA: controversies in the adjuvant therapy of high-grade gliomas. Oncologist 16:351-358 